\title{
A Second Order JFNK- Based IMEX Method for Single and Multi-Phase Flows
}

\section{The Sixth International Conference on Computational Fluid Dynamics}

\author{
Samet Kadiolgu \\ Dana Knoll \\ Mark Sussman \\ Richard Martineau
}

\section{July 2010}

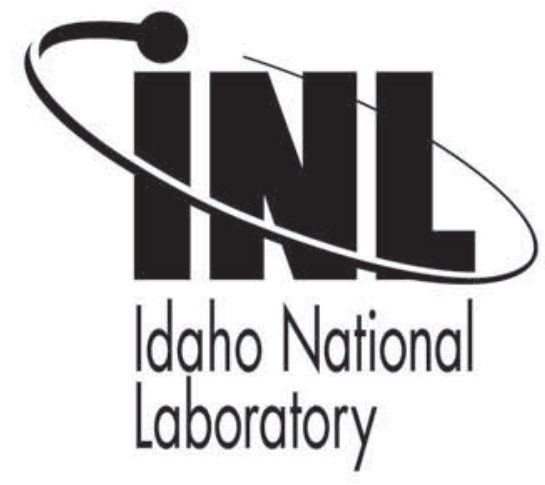

This is a preprint of a paper intended for publication in a journal or proceedings. Since changes may be made before publication, this preprint should not be cited or reproduced without permission of the author. This document was prepared as an account of work sponsored by an agency of the United States Government. Neither the United States Government nor any agency thereof, or any of their employees, makes any warranty, expressed or implied, or assumes any legal liability or responsibility for any third party's use, or the results of such use, of any information, apparatus, product or process disclosed in this report, or represents that its use by such third party would not infringe privately owned rights. The views expressed in this paper are not necessarily those of the United States Government or the sponsoring agency. 


\title{
A Second Order JFNK-based IMEX Method for Single and Multi-phase Flows
}

Samet Kadioglu, Dana Knoll, Mark Sussman, and Richard Martineau

\begin{abstract}
We present a second order time accurate IMplicit/EXplicit (IMEX) method for solving single and multi-phase flow problems. The algorithm consists of a combination of an explicit and an implicit blocks. The explicit block solves the non-stiff parts of the governing system whereas the implicit block operates on the stiff terms. In our self-consistent IMEX implementation, the explicit part is always executed inside the implicit block as part of the nonlinear functions evaluation making use of the Jacobian-free Newton Krylov (JFNK) method [7]. This leads to an implicitly balanced algorithm in that all non-linearities due to the coupling of different time terms are consistently converged. In this paper, we present computational results when this IMEX strategy is applied to single/multi-phase incompressible flow models.
\end{abstract}

Samet Kadioglu

Idaho National Laboratory, Fuels Modeling and Simulation Department, P.O. Box 1625, MS 3840, Idaho Falls, ID, 83415, USA, e-mail: samet.kadioglu@inl.gov

Dana Knoll

Los Alamos National Laboratory, Theoretical Division (T-3), P.O. Box 1663, MS B-216, Los Alamos, NM, 87545, USA, e-mail: nol@lanl.gov

Mark Sussman

Florida State University, Department of Mathematics, 208 Love Building, Tallahassee, FL, 32306, USA, e-mail: sussman@math.fsu.edu

Richard Martineau

Idaho National Laboratory, Fuels Modeling and Simulation Department, P.O. Box 1625, MS 3860, Idaho Falls, ID, 83415, USA, e-mail: richard.martineau@inl.gov 


\section{Introduction}

The IMplicit/EXplicit (IMEX) time integration technique has been increasingly used in physical applications that consist of multiple physics or exhibit multiple time scales $[1,9,2,6,4,5]$. In general, the governing equations for these kinds of applications consist of stiff and non-stiff terms. A typical IMEX method separates the stiff and non-stiff parts of the governing system and employs an explicit discretization (explicit block) that solves the non-stiff part and an implicit discretization (implicit block) that solves the stiff part of the problem. In a classic IMEX implementation $[1,9,2]$, the time operators are splitted such a way that the explicit and implicit algorithm blocks are executed independent of each other resulting in nonconverged non-linearities therefore time inaccuracies (order reduction to the first order in time accuracy for certain models is often reported $[2,4])$. On the other hand, in our self-consistent IMEX implementation, the explicit block is always solved inside the implicit block as part of the nonlinear functions evaluation making use of the Jacobian-Free Newton Krylov (JFNK) method [7]. In this way, there is a continuous interaction between the two algorithm blocks meaning that the improved solutions (in terms of time accuracy) at each nonlinear iteration are immediately felt by the explicit block and the improved explicit solutions are readily available to form the next set of nonlinear residuals. This continuous interaction between the two algorithm blocks results in an implicitly balanced algorithm in that all the nonlinearities due to the coupling of different time terms are consistently converged. In other words, we obtain an IMEX method that can maintain the formal order of time accuracy of the numerical scheme.

We have applied this JFNK-based IMEX methodology to a multi-physics problem that couples a neutron diffusion model to a thermally driven mechanics model to simulate transient behavior of fast burst reactors [6]. We have also considered multiple time scale flow models that consist of the compressible Euler equations plus very stiff heat conduction and/or source terms to solve radiation hydrodynamics problems $[4,5]$. For all of these applications, we have successfully showed demonstrated second order time convergence. In this paper, we consider incompressible single and multi phase flow models. In particular, the single-phase model consists of the incompressible Navier-Stokes equations and the multi-phase model considers the incompressible Navier-Stokes equations written in terms of the level set function plus the level set interface advection equation [11]. The JFNK-based IMEX method is applied to the single-phase flow model in the following manner. The hyperbolic terms of the flow equations (momentum advection terms except the pressure gradients) are solved explicitly exploiting the well understood explicit schemes. On the other hand, an implicit strategy is employed for the non-hyperbolic terms (viscous and pressure terms). In the multi-phase case, the IMEX method solves the momentum advection plus the interface advection terms explicitly and the viscous plus the stiff surface tension force terms implicitly. The implicit/explicit time integrations are carried out in a similar self-consistent fashion (refer to the first paragraph). 


\section{Governing Equations}

The single-phase flow model uses the following non-dimensional incompressible Navier-Stokes equations

$$
\begin{gathered}
\mathbf{u}_{t}+\mathbf{u} \cdot \nabla \mathbf{u}=-\nabla p+\frac{1}{R e} \Delta \mathbf{u}, \\
\nabla \cdot \mathbf{u}=0,
\end{gathered}
$$

where $\mathbf{u}=(u, v)$ is the flow velocity, $p$ is the fluid pressure, and $R e$ is the nondimensional Reynolds number. When we solve multi-phase (gas-liquid) flow systems, we rewrite Eq. (1) in terms of the level set function $(\phi)$ and add surface and body forces, i.e,

$$
\mathbf{u}_{t}+\mathbf{u} \cdot \nabla \mathbf{u}=-\frac{\nabla p}{\rho(\phi)}+\frac{1}{\operatorname{Re} \rho(\phi)} \nabla \cdot\left[\mu(\phi)\left(\nabla \mathbf{u}+(\nabla \mathbf{u})^{T}\right)\right]+\mathbf{F}^{s}+\mathbf{F}^{g} .
$$

The interface motion is governed by the level set advection equation,

$$
\phi_{t}+\mathbf{u} \cdot \nabla \phi=0,
$$

where $\phi(\mathbf{x}, t)>0$ if $\mathbf{x} \in$ liquid, $\phi(\mathbf{x}, t)<0$ if $\mathbf{x} \in$ gas, and $\phi(\mathbf{x}, t)=0$ if $\mathbf{x} \in \Gamma$ where $\Gamma$ represents the gas-liquid interface, and is defined as the zero level set of $\phi$. The density and viscosity can be written in terms of the level set function as $\rho(\phi)=\rho_{g}[1-H(\phi)]+\rho_{l} H(\phi)$ and $\mu(\phi)=\mu_{g}[1-H(\phi)]+\mu_{l} H(\phi)$ where $\rho_{g}$ $, \rho_{l}, \mu_{g}$, and $\mu_{l}$ represent the gas density, liquid density, gas viscosity, and liquid viscosity respectively. $H(\phi)$ is the smoothed Heaviside function [11]. The term $\mathbf{F}^{s}=-[1 /(W e \rho(\phi))] \kappa(\phi) \nabla H(\phi)$ represents the forcing term due to surface tension with $\kappa(\phi)=\nabla \cdot(\nabla \phi /|\nabla \phi|)$ being the local mean curvature. More information about the derivation and the problem parameters for these equations can be found in [10].

\section{The JFNK-based IMEX Method}

Assume that we are solving the multi-phase model, then the Explicit Block of IMEX solves advective terms of Eq. (3) (e.g, $\mathbf{u}_{t}+\mathbf{u} \cdot \nabla \mathbf{u}=0$ ) plus Eq. (4) whereas the Implicit Block operates on the right hand side of Eq. (3) (e.g., $\mathbf{u}_{t}=r h s$ ) plus the divergence free constraint (Eq. (2)). Our explicit and implicit time discretizations are based on a second order Runge-Kutta and Crank-Nicolson methods [8]. Our spatial discretizations for the explicit step are based on a second order essentially non-oscillatory (ENO) scheme, and centered differencing for the implicit step [8]. 
We write our implicit discretization as a system of non-linear equations $(F(U)=0$ where $U=(u, v, p))$ and employ the JFNK method to solve it. More details about how to form $F(U)$ and the IMEX algorithm implementations can be found in [4]. We remark again that the explicit step is always solved inside the implicit loop as part of the JFNK's non-linear functions evaluation. This helps to converge the tightly coupled non-linear system. To increase the efficiency of the JFNK solver, we use the classic pressure projection methodology as the preconditioner. Then the resulting elliptic system is relaxed by a few algebraic multi-grid V-cycles.

\section{Numerical Results}

First, we present results from the single-phase flow calculations. We consider a test problem that consists of a pair of horizontal shear layers of finite thickness, perturbed by a small amplitude vertical velocity. Details about the problem settings can be found in [3]. Fig. 1 shows the time history of the numerical solutions calculated in a doubly-periodic unit square with a $256 \times 256$ mesh. In time, each shear layer evolves into a periodic array of large vortices with the shear layer between the rolls being thinned by the large straining field there. Eventually, these thinned layers wrap around the large rolls. The evolution of the top and bottom layers are mirror images of one another. Notice that the flow is highly transient throughout the simulation time, so it is a good test to check the numerical time accuracy of the scheme. We provide a time convergence analysis for the $X / Y$-velocities and pressure in Fig. 2. Fig. 2 shows the decrease in the $L_{2}$-norm of the time errors of the numerical solutions. It is clear from this figure that we have obtained second order time convergence for all flow variables. Fig. 2 also provides some insights about the quality of our spatial discretization. It is evident form Fig. 2 that the method resolves the complicated flow structure reasonably well.
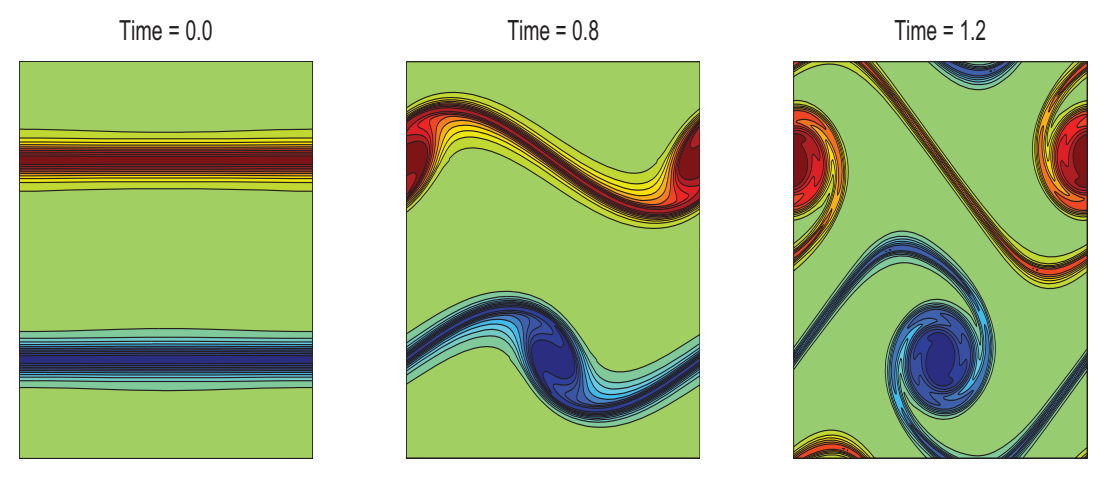

Fig. 1 Vorticity solutions from the thin shear layer problem on $256 \times 256$ mesh. 

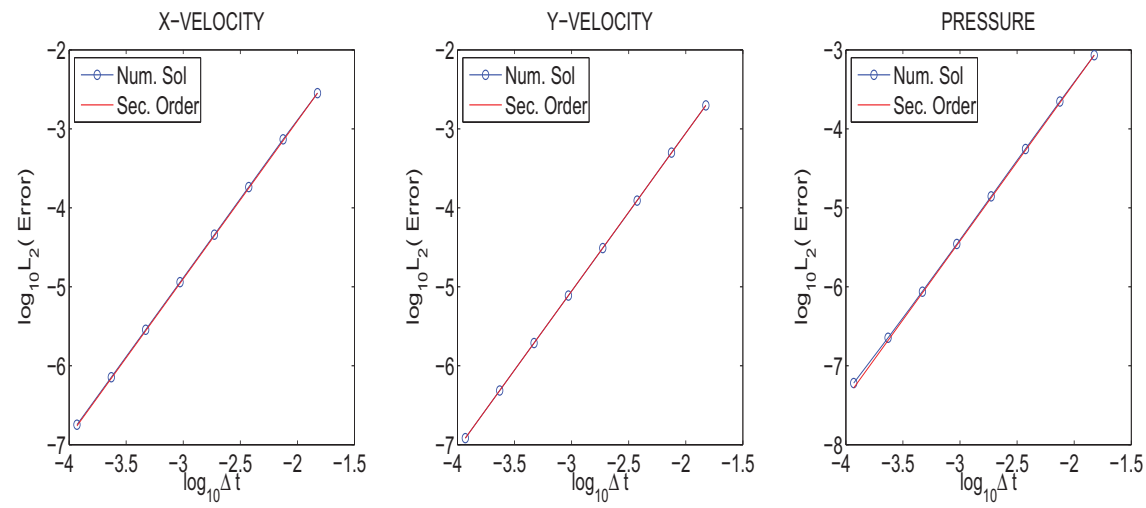

Fig. $2 L_{2}$ norm of the time errors for the thin shear layer problem on $256 \times 256$ mesh at time $=1.0$.

Now, we present results from the multi-phase flow calculations. The problem consists of a gas (air) bubble rising in an incompressible liquid (water). The initial bubble is located at $(x, y)=(1,1)$ in a $2 \times 4$ rectangular domain. Fig. 3 shows the time history of the bubble motion. The calculations are carried out with $100 \times 200$ mesh points. Notice that the bubble loses its circular shape in time due to surface forces effects. Also, we note that using a smoothed Heaviside function smears the interface into few cells (diffused interface capturing). Nonetheless, the density ratio can change from 1 to 1000 for this problem, therefore it is a challenging test for any multi-phase flow solver. Fig. 3 indicates that our method captures the inter-facial structure reasonably well. Moreover, our JFNK solver takes on average 3 Newton and 10 Krylov iterations. Table 1 compares the performance of our method to the CLSVOF method [11] for the same problem. Table 1 indicates that our method takes 11 times less number of time steps to reach the final time $=0.51$.
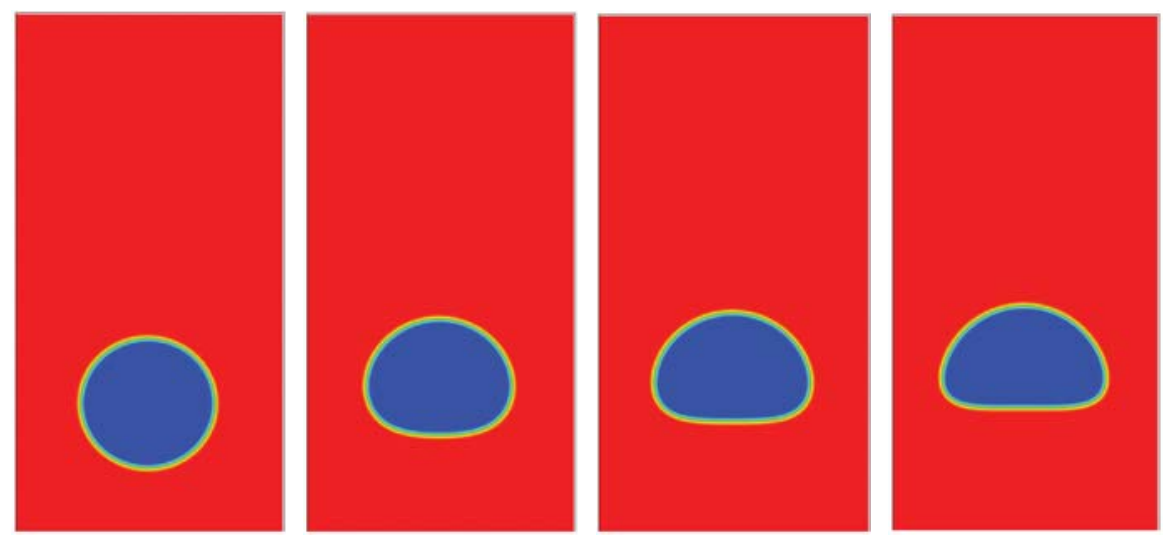

Fig. 3 Density at time $=$ (starting from top left) $0.00,0.35,0.43$, and 0.51 . 
Table 1 JFNK-based IMEX method versus CLSVOF method.

\begin{tabular}{|l|c|c|}
\hline & JFNK-based IMEX method & CLSVOF method \\
\hline Total \# time steps & 47589 & 523480 \\
\hline
\end{tabular}

\section{Conclusion}

We have presented a second order time accurate JFNK-based IMEX method for solving single and multi-phase flow problems. In our implicitly balanced IMEX method, all non-linearities due to the coupling of different time terms are consistently converged leading to second order time accurate calculations. The key feature of our method is that we carry out the explicit integrations as part of the non-linear functions evaluation within the JFNK framework. We have provided few examples to verify the accuracy and performance of our method.

Acknowledgements The submitted manuscript has been authored by a contractor of the U.S. Government under Contract No. DEAC07-05ID14517 (INL/CON-10-19312). Accordingly, the U.S. Government retains a non-exclusive, royalty-free license to publish or reproduce the published form of this contribution, or allow others to do so, for U.S. Government purposes.

\section{References}

1. U. M. Ascher, S. J. Ruuth, and B. T. R. Wetton. Implicit-explicit methods for time-dependent pde's. SIAM J. Num. Anal., 32:797-823, 1995.

2. J. W. Bates, D. A. Knoll, W. J. Rider, R. B. Lowrie, and V. A. Mousseau. On consistent time-integration methods for radiation hydrodynamics in the equilibrium diffusion limit: Low energy-density regime. J. Comput. Phys., 167:99-130, 2001.

3. J. B. Bell, P. Colella, and H. M. Glaz. A second order projection method for the incompressible navier-stokes equations. J. Comput. Phys., 85(2):257-283, 1989.

4. S. Y. Kadioglu and D. A. Knoll. A fully second order implicit/explicit time integration technique for hydrodynamics plus nonlinear heat conduction problems. J. Comput. Phys., 229:3237-3249, 2010.

5. S. Y. Kadioglu, D. A. Knoll, Robert B. Lowrie, and Rick M. Rauenzahn. A non-linearly consistent imex method for radiation hydrodynamics. J. Comput. Phys., Accepted, 2010.

6. S. Y. Kadioglu, D. A. Knoll, and C. Oliveria. Multi-physics analysis of spherical fast burst reactors. Nuclear Science and Engineering, 163:1-12, 2009.

7. D. A. Knoll and D. E. Keyes. Jacobian-free Newton Krylov methods: a survey of approaches and applications. J. Comput. Phys., 193:357-397, 2004.

8. R.J. Leveque. Finite Volume Methods for Hyperbolic Problems. Cambridge University Press , Texts in Applied Mathematics, 1998.

9. R. B. Lowrie, J. E. Morel, and J. A. Hittinger. The coupling of radiation and hydrodynamics. Astrophys. J., 521:432, 1999.

10. E. Olsson and G. Kreiss. A conservative level set method for two phase flow. J. Comput. Phys., 210:225-246, 2005.

11. M. Sussman and E.G. Puckett. A coupled level set and volume-of-fluid method for computing 3d and axisymmetric incompressible two-phase flows. J. Comput. Phys., 162:301-337, 2000. 